\title{
The Contact Resistance Force Relationship of an Intrinsically Conducting Polymer Interface
}

\author{
Yu-Zhi (Liza) Lam, Jonathan Swingler, and John W. McBride
}

\begin{abstract}
Investigations on contact connector materials for different applications such as in the automotive industry have focused toward the increasing interest of using conducting polymers, as compared to conventional metallic contacts. The aim is to achieve overall improvements in performance as well as cost effectiveness. Currently, extrinsic conducting polymers (ECPs) are employed as conductive coats or adhesives at contact interfaces. However, frictional abrasion within the metal doped polymer (ECP) causes fretting corrosion, which leads to instability in the contact resistance. To overcome this, intrinsically conducting polymers (ICPs) are explored. Hemispherical contact coatings were fabricated using poly(3,4-ethylenedioxythiopene) (PEDOT) or polyaniline/polyvinylchloride (PANI/PVC) commodity blends. Contact resistances were taken using four-wire resistance measurement techniques. The conductivities of in-house fabricated ICP contacts were found to be in the range of $10^{-2} \mathrm{~S} \cdot \mathrm{cm}^{-1}$. The response relating the change of contact resistance under varying compression force appeared to be repeatable with minimum deviation of $2 \%$. The surface profiles of the ICP contacts were also recorded by an optical confocal system. The initial investigation results presented in this paper were used to evaluate and validate the hypothesis of employing ICP contacts to eliminate or minimize wearing and fretting effects.
\end{abstract}

Index Terms-Contact connector, contact resistance, fretting, intrinsically conducting polymer (ICP), surface profile.

\section{INTRODUCTION}

A PPROXIMATELY 30 years ago, all carbon based polymers were rigidly regarded as insulators. This narrow perspective has rapidly changed as a new class of polymer known as intrinsically conducting polymer (ICP) or electroactive polymers are being discovered. Although this class is in its infancy, the potential applications of ICPs are relatively significant [1]. One of such applications is the automotive industry where metal terminals could be replaced by conducting polymer contacts. This would contribute toward improving the economical aspects of the overall electrical wiring system within automobiles by reducing the cost of materials as well as the weight, which directly affect the amount of energy being consumed.

In 1960s, the researchers at Plastics Research Laboratory of BASF focused on postulating new structural properties of

Manuscript received October 18, 2004. This work was supported through the European Commission "Growth" Programme, Research Project AUTOCON: "Investigations into integrated wiring and interconnecting of electrical and electronic components for intelligent systems," under Contract GIRD-CT01-00 588. This work was recommended for publication by Associate Editor M. Braunovic upon evaluation of the reviewers' comments.

The authors are with the School of Engineering Sciences, University of Southampton, Southampton S017 1BJ, U.K. (e-mail: lizalam@ mech.soton.ac.uk).

Digital Object Identifier 10.1109/TCAPT.2006.875903 polymers made by oxidative coupling [1]. This reaction included aromatic compounds and heterocycles. It was found that polyphenylene and polythiophene showed electrical conductivities of approximately $0.1 \mathrm{Scm}^{-1}$, which indicated the initial capability of conducting electricity. For references, copper has the conductivity of $0.596 \times 10^{6} \mathrm{Scm}^{-1}$ [2].

Different polymers and polymer derivatives, doped with a weak oxidation agent or reducing agent resulting in various conjugated polymers, had brought about the understanding of charge storage and charge transfer mechanisms [3]. However, the main problems of these polymers are their processibility and stability. The development of highly conducting polymers with good stability and acceptable processing attributes are the main focuses of many recent research works.

In electronics and electrical systems, the use of polymer connectors is emerging to take on conventional metallic contacts as cost effective alternatives. Conducting polymers doped with metal particles, or better known as extrinsic conducting polymer (ECP) contacts have advantages such as low cost; easy fabrication methods and generally good thermal and mechanical properties. Furthermore, ECPs as alternative contact materials also could reduce the use of precious metals and improve reliable by minimizing the effects of fretting [4]. However, due to the doped metal particles, some degree of abrasion or fretting is often experienced at the particle surface interfaces within the ECPs. This leads to possible deformation of contact surfaces and hence variations in resistance values could affect the overall system over periods of usage.

The main objective here is to reduce fretting further and yet incorporate the advantages of ECPs by studying the possibilities of employing intrinsically conducting polymers (ICPs) in place of ECPs for applications involving electrical contacts. The hypothesis made for overcoming the abrasive effects of ECPs is mainly attributed to the elastic property of ICPs. With this, ICPs retain the ability to conduct and possess the capability of absorbing the effects of external forces at the contact interface.

This paper presents an initial investigations made on two types of ICPs namely poly(3,4-ethylenedioxythiophene) (PEDOT) and polyaniline (PANI). The ICPs were doped with different ratios of solvents and prepared under certain conditions to achieve improvements in the mechanical structure, processibility and conductivity. Experiments were carried out to measure the contact resistances using four-wire resistance measurement techniques while subjected to a normal force. To observe and compare the physical changes of the ICPs, the surface distributions of the samples were taken before and after the experiments. 
TABLE I

SUMMARY OF ICPS' CHARACTERISTICS

\begin{tabular}{|l|c|c|}
\hline \multicolumn{1}{|c|}{ Polymer } & Conductivity $\left(\mathrm{Scm}^{-1}\right)$ & Percentage w.r.t. copper \\
\hline PPy & Up to 10 & $\sim 0.001 \%$ \\
\hline PANI & Up to 400 & $\sim 0.04 \%$ \\
\hline PEDOT & 0.01 to 100 & $\sim 0.01 \%$ \\
\hline
\end{tabular}

\section{OPERATIONAL PRINCIPLE OF ICP}

Intrinsically conducting polymers (ICPs) belong to a class of organic materials with unique electronic properties such as electric conductivity, electrochromism, and electroactivity. Conjugated electrons in the backbone of their macromolecules are responsible for these properties. Due to their high electrical properties, ICPs are intensively investigated for application in electronics, microelectronics, optoelectronics mainly for areas in aerospace and automobile industries [5]. Among the most promising applications of the ICPs are corrosion protection [6], solid-state charge storage devices [6], electromagnetic screens [7], antistatic coatings and gas separation coatings [8]. However, poor mechanical properties, environmental sensitivity, moderate stability of electrical properties with temperature significantly limit the industrial applicability of ICPs. Different values of the electrical conductivity (as summarized in Table I) and mechanical properties are exhibited by various ICPs. Polythiophenes (PTPh), polypyrrole (PPy), and polyaniline (PANI) are generally the most studied conducting polymers.

Poly(3,4-ethylenedioxythiophene) (PEDOT) is a relatively new member in the conducting polymer family and displays interesting properties including low half-wave potential and band gap, and relatively good electrochemical, air and thermal stability of its electrical properties as compared to other polythiophenes.

\section{A. Principle of Conduction}

The method of conduction using "band theory" was initially applied to conducting polymers. It was found that the polymer could more efficiently lower its energy by bond alteration via doping, hence making it similar to a high energy gap semiconductor [9]. In order to completely understand the conductivity of polymers, the way in which charge is stored along the polymer chain and its effect plays an important role.

The polymer usually store charges in two ways. In an oxidation process, it could either lose an electron from one of the bands or it could localize the charge over a small section of the chain. Localizing the charge causes a local distortion and an effective change in geometry, which reduces some the energy of the polymer. The generation of this local geometry decreases the ionization energy of the polymer chain and increases its electron affinity, hence, increasing its ability to accommodate newly formed charges. A similar scenario occurs for a reductive process. The main criterion is the ability to oxidize or reduce the polymer without lowering its stability.

The combination of a charge site and a free radical is known as a polaron. Upon further oxidation the free radical of the polaron is removed, creating a new spinless defect called a bipolaron as shown in Fig. 1. For a very heavily doped polymer
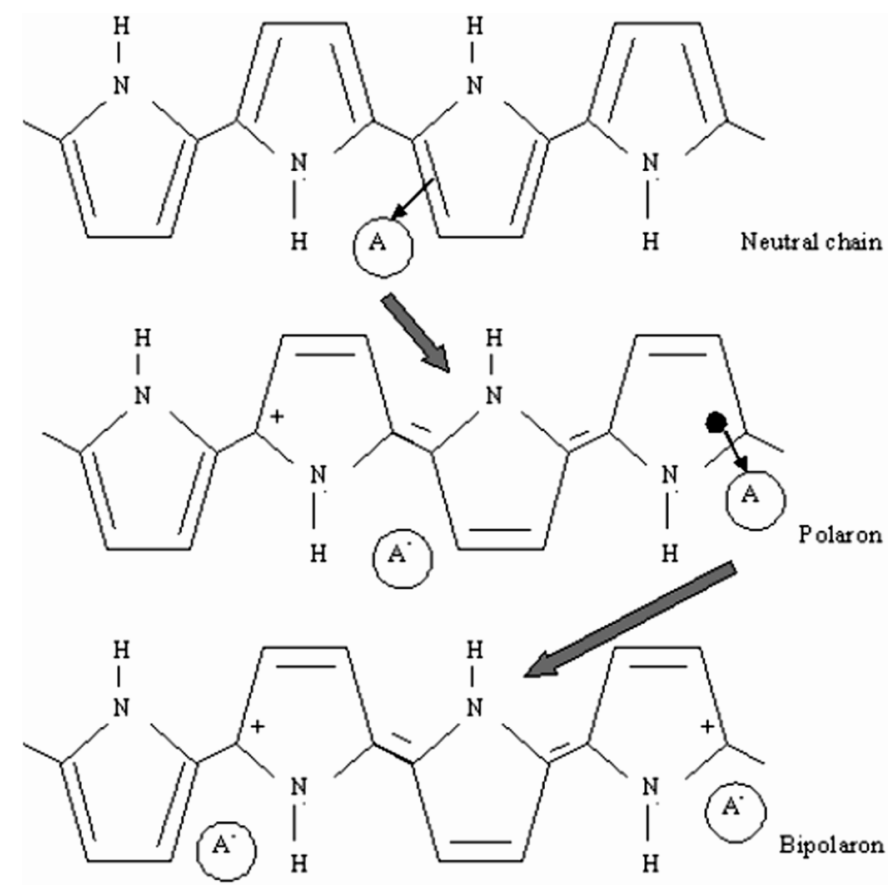

Fig. 1. Formation of polaron and bipolaron.

it is conceivable that the upper and the lower bipolaron bands will merge with the conduction and the valence bands respectively to produce partially filled bands and metal-like conductivity. Charge transfer between the conducting domains occurs by thermally activated hopping or tunnelling.

\section{B. Stability and Processibilty}

Extrinsic stability is related to vulnerability to external environmental agent such as oxygen, water, peroxides. This is determined by the polymers susceptibility of charged sites to attacks by nucleophiles, electrophiles and free radicals. Protection can be given by a stable coating. However, many conducting polymers degrade over time even in dry and oxygen free environment. This leads to intrinsic instability and is likely to be caused by irreversible chemical reaction between charged sites of polymer and either the dopant counter ion or the p-system of an adjacent neutral chain, which produces an $\mathrm{sp}^{3}$ carbon by breaking the conjugation. The charge sites become unstable due to conformational changes in the polymer backbone. Conjugated polymers may be processed by a number of different techniques including cationic, anionic [10], radical chain growth [11], coordination polymerization [12], step growth polymerization or electrochemical polymerization [5].

\section{EXPERIMENTAL DETAILS}

\section{A. PEDOT and Doped Derivatives}

PEDOT doped with poly(4-styrenesulfonate) (PSS) has received much attention over the last decade [13]. PEDOT/PSS using $\mathrm{H}_{2} \mathrm{O}$ as a pristine solvent is optically transparent and a hole transport material. The commercially available form of PEDOT/PSS is known as BAYTRON P from Bayer AG and the molecular structure is shown in Fig. 2. It is a dispersion of conducting polymer within an aqueous solution. 


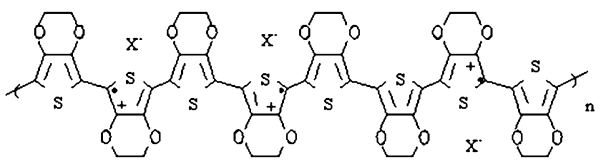

Fig. 2. PEDOT/PSS structure.

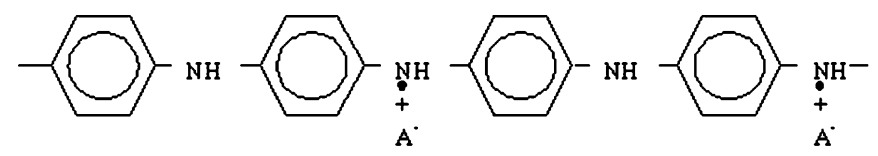

Fig. 3. PANI (emeraldine salt) structure.

The weight ratio of PEDOT to PSS is 1:1.6 with the mixture diluted in $\mathrm{H} 2 \mathrm{O}$. BAYTRON P is a material that is easily manipulated in terms of fabricating conductive coats and films. It was found by Joo et al. that the conductivity of pristine PEDOT/PSS is approximately $0.8 \mathrm{Scm}^{-1}$ [13]. He also enhanced the conductivity up to $80 \mathrm{Scm}^{-1}$ by mixing dimethyl sulfoxide (DMSO), tetrahydrofuran (THF) and dimethylformamide (DMF) respectively as solvents. Initial in-house tests have shown that using PEDOT/PSS with DMSO or THF as conductive coatings tended to be more brittle and susceptible to damage when pressure was applied.

For this paper, the key issue lies in the introduction of different weight ratios of dimethylformamide (DMF) into PEDOT/PSS in order to increase the conductivity of the overall polymer based on the in-house fabrication methods used to generate ICP contact surfaces.

\section{B. Polyaniline and Blends}

Polyaniline (PANI) has been employed in a wide variety of electronic applications such as light emitting diodes, semiconductor devices and blend with insulative host polymer to impart electrical conductivity [14]. Generally, depending on different fabrication and processing methods, the electrical conductivity could range from $10^{-8}$ to $10^{2} \mathrm{Scm}^{-1}$. A reduction in structural defect will increase the conductivity. PANI easily switches from an insulator (emeraldine base) to a conductor (emeraldine salt) as a function of $\mathrm{pH}$ levels.

For this investigation, a form of polyaniline complexed (doped) with proprietary organic sulfonic acid (emeraldine salt, Catalogue Number 42 832-9 from Aldrich) is employed as an inherently conducting polymer. It is a black powder and its chemical composition is illustrated in Fig. 3.

The PANI powder is insoluble in many solvents hence fabricating conductive pellets or coating for electrical contacts proves to be challenging as the structure remains brittle after the fabrication process. To overcome this problem, PANI is mixed into polyvinyl chloride (PVC), an insulating polymer using tetrahydrofluran (THF, Catalog Number 87369 from FLUKA) as a solvent. This way, the mechanical structure takes on the insulating polymer while maintaining some conductivity property within the blend [15].

\section{Experiment Details}

Prior to the experiments, the surface profiles of the ICP-coated ECP columns and tin-lead PCB tracks were

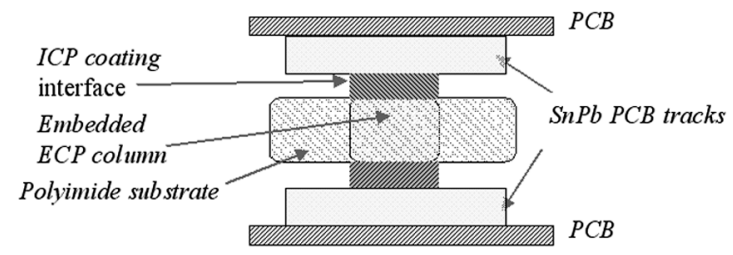

Fig. 4. Setup "sandwiched” configurations with ICP-coated ECP sample.

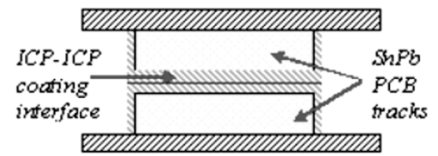

(a)

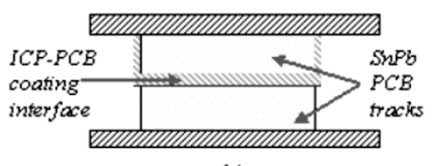

(b)
Fig. 5. Setup "sandwiched" configurations (a) ICP/ICP interface contact sample and (b) ICP/PCB interface contact sample.

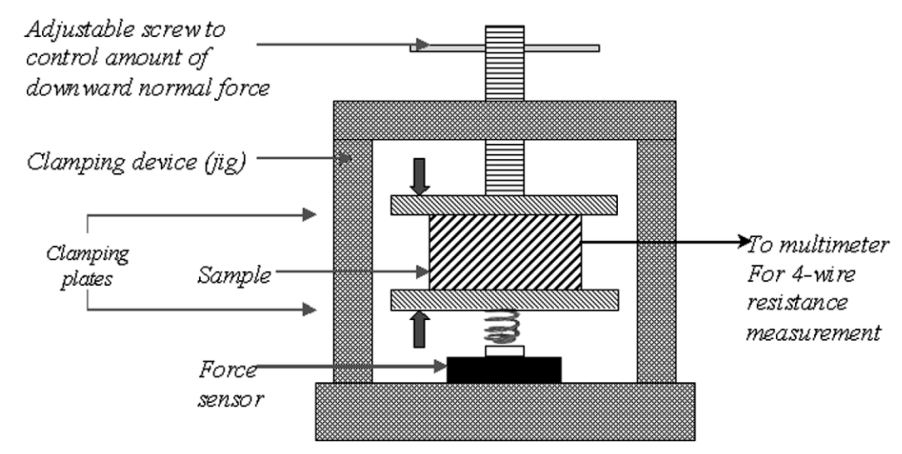

Fig. 6. Clamping setup for experiments using force exertion.

acquired using a confocal system (TaiCaan Technologies Ltd.). At the end of the experiments, the same tested samples were then subjected to the same optical measurements. This is to facilitate the investigations of the physical effects such as deformation or abrasive traces by comparing the untested and tested samples. The setups for the each contact sample were based on three basic configurations as depicted in the schematics (not drawn to scale) of Figs. 4 and 5.

All SnPb PCB tracks were joined to solder terminals where external connections were made for four-wire resistance measurement using the Keithley 2000 multimeter. Samples with different ICPs in each "sandwiched" configuration were placed between the clamping devices as shown in Fig. 6. A force sensor (Model: FSG15N1A from Honeywell micro switch sensing and control) was placed under the sample to record the force exerted by the clamp.

The adjustable screw of the clamping jig was set to exert a fixed amount of force across the "sandwiched" configuration. Each of the 60 recordings for the contact resistances was taken at 45-s interval and carried out in ambient conditions. With accordance to the industrial specifications [16] for the general ECP columns, the compression force should be approximately $10 \mathrm{~N}$. Hence in the experiments, the contact resistances of the samples were measured for different values of forces, which ranged between $5 \mathrm{~N}$ and $10 \mathrm{~N}$. It was noted that the exerting forces of less than $5 \mathrm{~N}$ were not used due to the large resistance values (in the mega-ohm range) measured. 


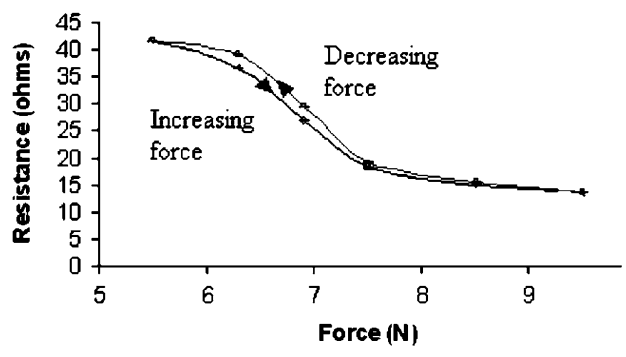

Fig. 7. Contact resistance measured against varying force for the original PEDOT/PSS-coated ECP column.
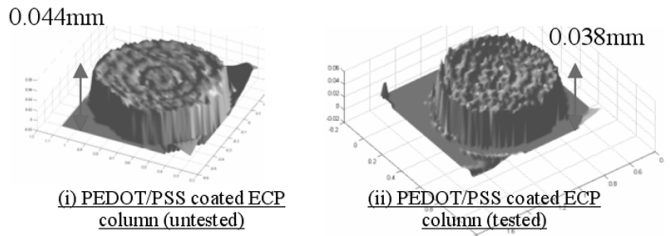

Fig. 8. Surface profiles of PEDOT/PSS-coated ECP columns (untested and tested samples, over $2 \mathrm{~mm} \times 2 \mathrm{~mm}$ area).

\section{RESULTS}

1) Raw Data: There were altogether six different ICP materials used namely original PEDOT/PSS, PEDOT/PSS:DMF (5:1), PEDOT/PSS:DMF (4:1), PEDOT/PSS:DMF (3:1), PEDOT/PSS:DMF (2:1) and PEDOT/PSS:DMF (1:1). Each ICP-coated ECP column (as depicted in Fig. 4) was placed in the clamping jig as shown in Fig. 6 and subjected to a constant calibration force of approximately $8.5 \mathrm{~N}$. Using the four-wire resistance technique, the averaged contact resistance of the original PEDOT/PSS-coated ECP column was measured to give $15.10 \Omega \pm 0.79 \%$. This measurement was repeated for each of the ECP columns coated with the rest of the five remaining derivatives. The averaged contact resistances were $12.63 \Omega \pm 4.51 \%$, $10.28 \Omega \pm 2.82 \%, 3.75 \Omega \pm 3.74 \%, 34.44 \Omega \pm 11.09 \%$ and $599.42 \Omega \pm 3.65 \%$ for the PEDOT/PSS:DMF ratios of (5:1), (4:1), (3:1), (2:1), and (1:1), respectively.

Each sample was then subjected to a varying compression force from $5.5 \mathrm{~N}$ to $9.5 \mathrm{~N}$. Fig. 7 shows the results obtained for the PEDO T/PSS-coated ECP column. This procedure was repeated for the five remaining derivative samples of different weight ratios. In order to analyze the effects of the compression force on the samples, the final stage of the investigation was to measure the corresponding surface profiles before and after the compression force experiment. Fig. 8 shows the untested and tested PEDO T/PSS-coated sample's surface profiles. It was seen that there was a reduction in the height of the contact after the compression force experiment. This indicated a change in contact dimensions in terms of the cross-sectional area and the length of contact interface. Fig. 9 summarizes the approximated change in height for the samples coated with PEDO T/PSS and the derivates. The height of the ECP column corresponded to the length of contact interface. It was found that more significant differences in the change of length (or height) of ECP column contacts were observed in the samples with higher DMF content.

2) Analysis of ICP-Coated ECP Columns: The conductivities $(\sigma)$ of different ICPs are calculated from the results obtained

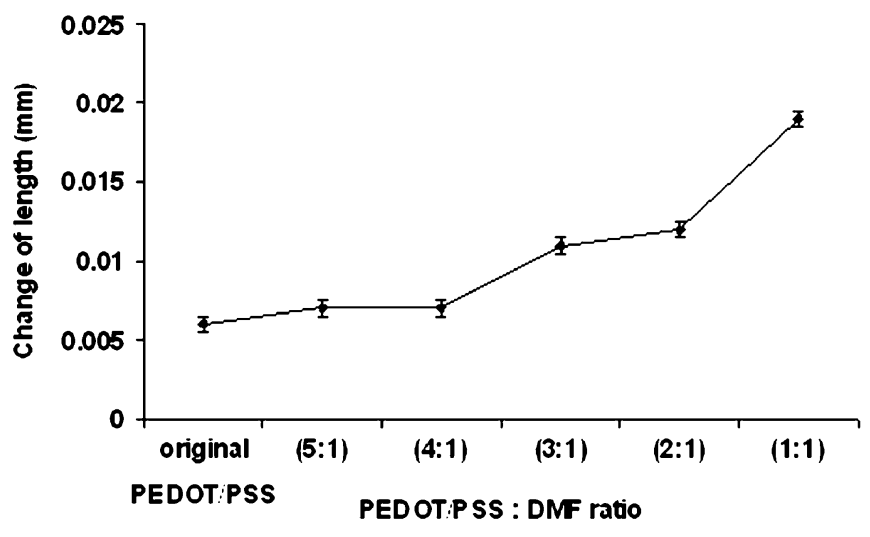

Fig. 9. Change of contact length for different PEDOT/PSS:DMF ratios.

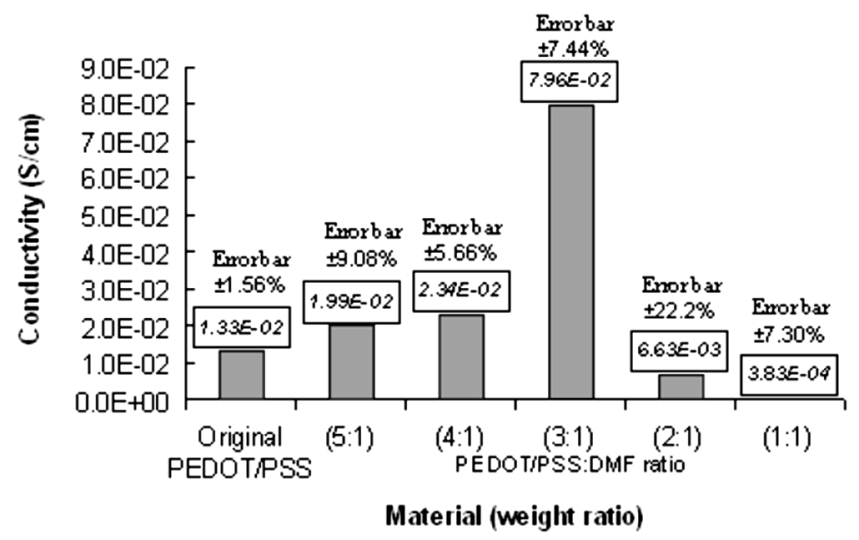

Fig. 10. Conductivity of PEDOT/PSS (:DMF)-coated ECP columns.

by the reciprocal of the four-wire resistance measurement $(R)$ multiplied by the form factor, given by the ratio of contact length $(l)$ and contact area $(A)$ as shown in

$$
\sigma=\frac{l}{A \times R} .
$$

Coating ICPs onto ECP column surfaces allowed for more consistent form factors due to the manageable size and processibility. Therefore, in the evaluation of the conductivity for in-house fabricated contacts coated with PEDOT/PSS and the derivatives as summarized in Fig. 10, the averaged values of contact resistances $(R)$ measured from the ICP-coated ECP columns with exerting forces larger than $8.5 \mathrm{~N}$ were used. The contact length and contact areas were $0.001 \mathrm{~cm}(\sim 10 \mu \mathrm{m})$ and $0.005027 \mathrm{~cm}^{2}$, respectively. The contact resistance was measured effectively across two ICP interfaces and a "sandwiched" ECP column (in series). The conductivity of an uncoated ECP column was relatively high at approximately $200 \mathrm{Scm}^{-1}$, which produced resistance values in the range of a few hundred $\mathrm{m} \Omega$. Compared to the conductivity of the ICP-coated ECP column in the minimum range of $10^{-2} \mathrm{Scm}^{-1}$, the contribution of the ECP columns to the overall conductivity could be neglected in order to simplify the evaluation.

3) Variation of Form Factor (1/A Ratio) With Contact Force: The "sandwiched" PEDOT- and PEDOT/DMF-coated ECP columns (Fig. 4) were placed individually in the setup as depicted in Fig. 6. They were subjected to several cycles of compression forces, which increased from $5.5 \mathrm{~N}$ to $9.5 \mathrm{~N}$ and 

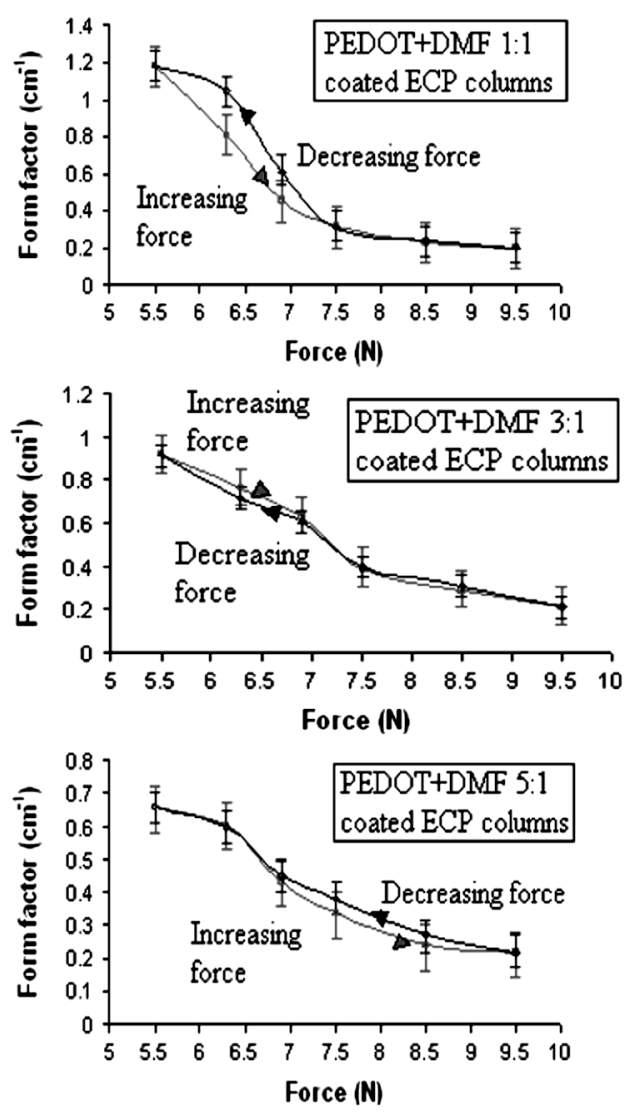

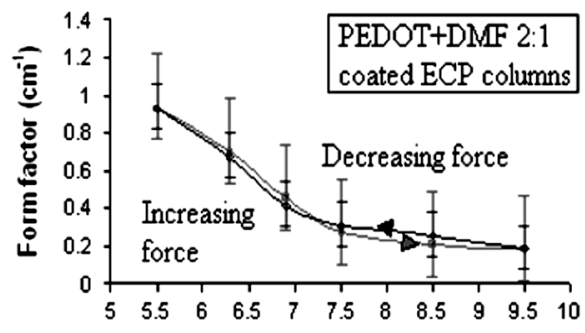

Force (N)

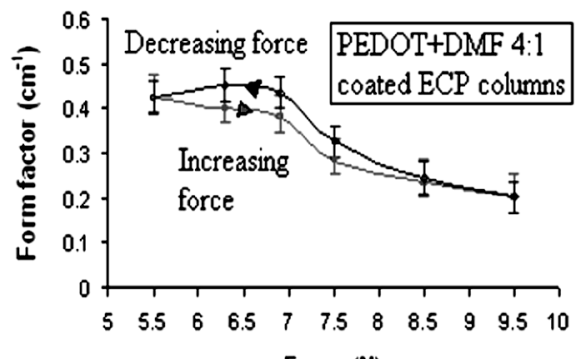

Force (N)

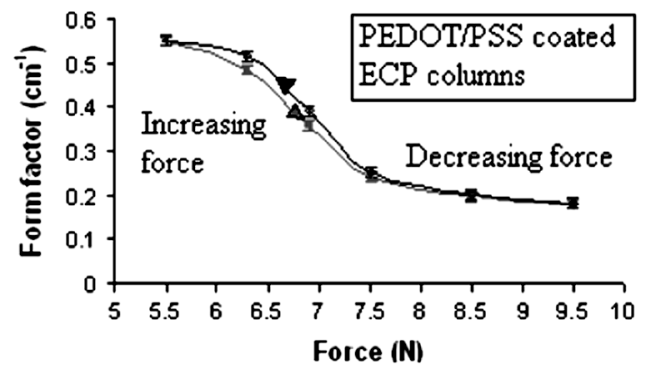

Fig. 11. Form factor of different ICPs (coated onto ECP columns) with respect to change of force.

decreased at the same intervals in the normal direction. The force of $5.5 \mathrm{~N}$ was the minimum amount required to obtain a reasonable contact resistance measurement. The contact resistances of the PEDOT/PSS-coated ECP columns were measured at each interval as shown previously in Fig. 7.

The data in Fig. 10 is used to predict the form factor change in the samples with contact force, assuming that conductivity is constant. The form factor (given by the $l / A$ ratio) was obtained by multiply the conductivity of the PEDOT/PSS (as given in Fig. 10) with the resistance measured. This procedure was carried out to the remaining five derivatives with different ratios of PEDOT/PSS and DMF. Fig. 11 shows the changes of form factors with respect to varying forces. It was observed that as the amount of DMF increased in the ICP mixture, the measurements became less repeatable over several cycles of changing forces.

4) Analysis of ICP-Coated PCB Tracks: Another series of investigations were carried out using the configurations of ICPcoated PCB tracks (refer to Fig. 5). Due to the coating of a larger surface area as compared to that of the ECP column, it was difficult to control the consistency of ICP layers in terms of thickness and flatness. The contact resistances were measured using the four-wire measurement technique when each sample was subjected to the calibration force of $8.5 \mathrm{~N}$. Table II shows the averaged contact resistances and the respective dimensions of different materials for the configuration of two "sandwiched" ICPcoated PCBs (PCB/ICP-IC/P/PCB interface). Table III summarizes the results for the configuration of an ICP-coated PCB
TABLE II

MEASUREMENTS FOR THE DRIED ICP-DRIED ICP INTERFACE

\begin{tabular}{|l|c|c|}
\hline $\begin{array}{c}\text { Material with Contact } \\
\text { Length of 0.024cm ( } \pm \text { 4.17\%) }\end{array}$ & Area $\left(\mathrm{cm}^{2}\right)$ & $\begin{array}{c}\text { Measured Resistance } \\
(\mathrm{K} \Omega)\end{array}$ \\
\hline Original PEDOT/PSS & $0.000201( \pm 2.50 \%)$ & $13.027( \pm 8.96 \%)$ \\
\hline PEDOT:DMF (5:1) & $0.000201( \pm 2.50 \%)$ & $11.024( \pm 12.94 \%)$ \\
\hline PEDOT:DMF (4:1) & $0.000201( \pm 2.50 \%)$ & $9.920( \pm 12.60 \%)$ \\
\hline PEDOT:DMF (3:1) & $0.000201( \pm 2.50 \%)$ & $8.742( \pm 13.05 \%)$ \\
\hline PEDOT:DMF (2:1) & $0.000314( \pm 3.20 \%)$ & $26.531( \pm 14.87 \%)$ \\
\hline PEDOT:DMF (1:1) & $0.000314( \pm 3.20 \%)$ & $435.204( \pm 16.46 \%)$ \\
\hline
\end{tabular}

pressed against another PCB track, forming a PCB/ICP-PCB interface. With the information presented in Tables II and III, the calculated conductivities of the respective configurations are plotted in Fig. 12.

The samples were then subjected to varying normal forces ranging from $6.3 \mathrm{~N}$ to $9.5 \mathrm{~N}$. In this case, $6.3 \mathrm{~N}$ was the minimum force required to get reasonable measurable values. It can be seen that the conductivity increased as the amount of DMF in the derivative increased, giving the optimum result with the PEDOT/PSS:DMF ratio at 3:1. As the amount of DMF increased further, the conductivity decreased thereafter. This trend was also observed with the samples of ICP-coated ECP columns (refer to Fig. 10). The conductivities of the ICP-PCB track interfaced structure and that of the ICP-coated ECP columns were generally comparable with the averaged 
TABLE III

MEASUREMENTS FOR THE DRIED ICP-PCB TRACK INTERFACE

\begin{tabular}{|l|c|c|}
\hline $\begin{array}{c}\text { Materinl with Contact } \\
\text { Length of } 0.012 \mathrm{~cm}( \pm 8.33 \%)\end{array}$ & Area $\left(\mathrm{cm}^{2}\right)$ & $\begin{array}{c}\text { Measured Resistance } \\
(\mathrm{K} \Omega)\end{array}$ \\
\hline Original PEDOT/PSS & $0.000227( \pm 2.20 \%)$ & $5.152( \pm 3.21 \%)$ \\
\hline PEDOT:DMF (5:1) & $0.000531( \pm 1.88 \%)$ & $1.498( \pm 2.44 \%)$ \\
\hline PEDOT:DMF (4:1) & $0.001257( \pm 0.80 \%)$ & $0.438( \pm 14.50 \%)$ \\
\hline PEDOT:DMF (3:1) & $0.000804( \pm 1.24 \%)$ & $0.204( \pm 25.00 \%)$ \\
\hline PEDOT:DMF (2:1) & $0.000201( \pm 2.50 \%)$ & $11.516( \pm 6.48 \%)$ \\
\hline PEDOT:DMF (1:1) & $0.001964( \pm 1.02 \%)$ & $18.452( \pm 10.01 \%)$ \\
\hline
\end{tabular}

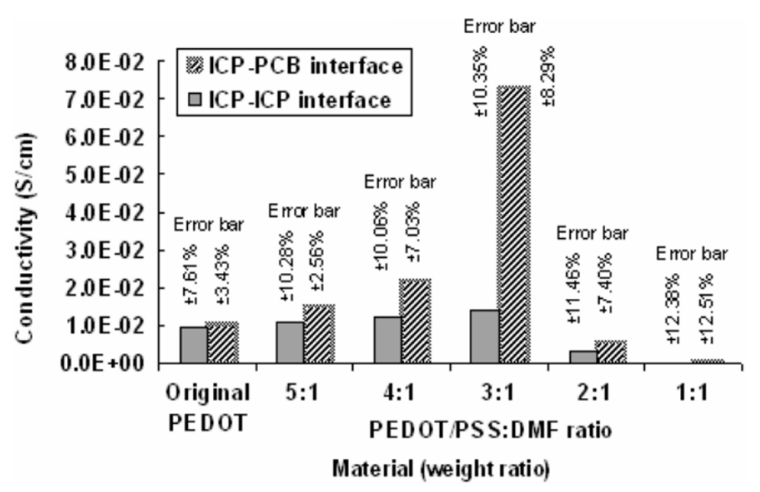

Fig. 12. Conductivity of ICP-coated PCB tracks.

variation of $16.2 \%$. On the other hand, the ICP-ICP interfaced structure have relatively larger conductivities with minimum and maximum variations of $30.9 \%$ and $82.8 \%$ respectively for various mixtures of PEDOT/PSS and DMF ratios as compared to the ICP-coated ECP columns.

As different ICP-coated surfaces have inconsistent dimensions, it was important to note that the values of the contact resistance were not valid representations hence cross-comparison among different materials were not encouraged. Nonetheless, from the results, it was found unanimously that as force increased, contact resistances decreased for all different PEDOT/PSS and the derivatives.

\section{A. PANI and PVC Blends}

1) Raw Results: There were altogether five different ratios of PANI and PVC blends used namely (4.73:1), (4.33:1), (4:1), (2.89:1), and (2.6:1). At the constant calibration force of approximately $8.5 \mathrm{~N}$, the contact resistance of (4.73:1) PANI/PVC blend-coated ECP column was found to be $728 \Omega \pm 8.54 \%$. This measurement was repeated for each of the ECP columns coated with the rest of the four remaining blends. The contact resistances were $5676.36 \Omega \pm 1.93 \%, 2959.95 \Omega \pm 2.46 \%, 6367.76 \Omega \pm 3.83 \%$ and $41283.86 \Omega \pm 13.19 \%$ for the PANI:PVC blend ratios of (4.33:1), (4:1), (2.89:1), and (2.6:1), respectively.

2) Analysis of ICP-Coated ECP Columns: Fig. 13 summarizes the conductivities calculated from the contact resistance measurements of various PAN I/PVC blend-coated ECP columns. The PANI/PVC blends had paste-like textures, which incurred difficulties in obtaining coats of similar dimensions under the in-house fabrication conditions. In another words,

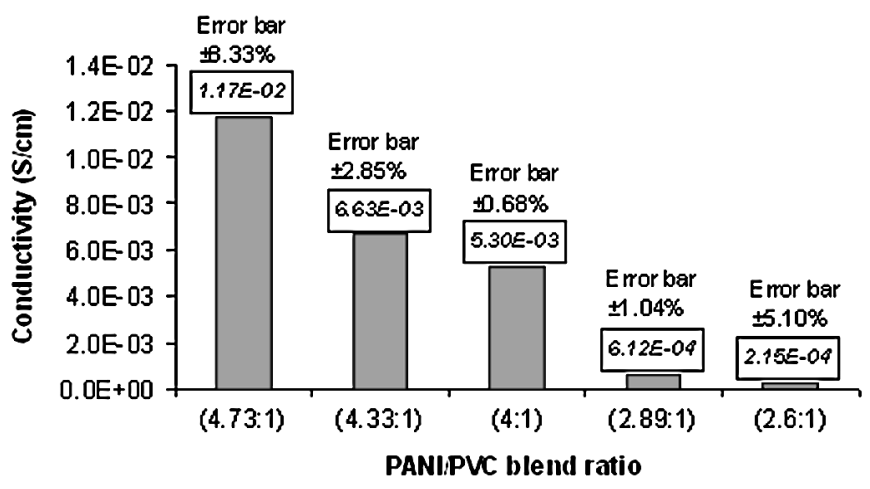

Fig. 13. Conductivity of different PANI/PVC blends.

the contact areas and contact lengths of each interface were different. Nonetheless, it was observed that as the amount of PVC increased in the blend, the conductivity decreased as the insulative property becomes more dominant.

The "sandwiched" PANI/PVC blend-coated ECP columns (as in Fig. 4) were placed in the setup as depicted in Fig. 6. They were subjected to several cycles of compression forces, which increased from $7.0 \mathrm{~N}$ to $9.8 \mathrm{~N}$ and decreased at the same intervals in the normal direction. The contact resistances were measured at each interval. In this experiment, reasonable measurements were only available at the minimum force of $7 \mathrm{~N}$. The form factor ( $l / A$ ratio) of each blend was obtained by multiply the conductivity of the material with the corresponding resistance measured. Fig. 14 shows the variation of form factor with respect to changing forces, including the error bars which are relatively small for all cases.

Generally, it was observed that hystereses were formed by the form factors taking the different paths of increasing and decreasing forces over the same intervals. At the maximum force of $9.8 \mathrm{~N}$, measurements for each blend type appeared to converge to a similar point. At the minimum force of $7 \mathrm{~N}$, the structure relaxed and the PANI/PVC blend could return approximately to its original dimensions as suggested by the convergence of the form factor value over several cycles. It was noted that when the amount of insulating polymer in the blend was small as in the 4.73:1 (PANI:PVC) blend, the ability to obtain a similar form factor after each force-varying cycle was less possible. Despite the hysteresis effect, the measurements also revealed relatively good repeatability of form factors at different intervals of compression forces as the amount of DMF in the blend increased. In order to analyze the effects of the compression force on the samples, the corresponding surface profiles before and after the compression force experiment were measured. The most significant variation in contact length appeared to be found for the samples coated with PANI/PVC (4.73:1). Less variation was noted for the samples coated with blends containing higher PVC contents.

3) Analysis of ICP-Coated PCB Tracks: Table IV summarizes the averaged contact resistances and the respective dimensions of different blend ratios for the configurations of two "sandwiched" ICP-coated PCBs (PCB/ICP-IC/P/PCB interface) as well as an ICP-coated PCB pressed against a PCB track (PCB/ICP-PCB interface). The lengths and respective 

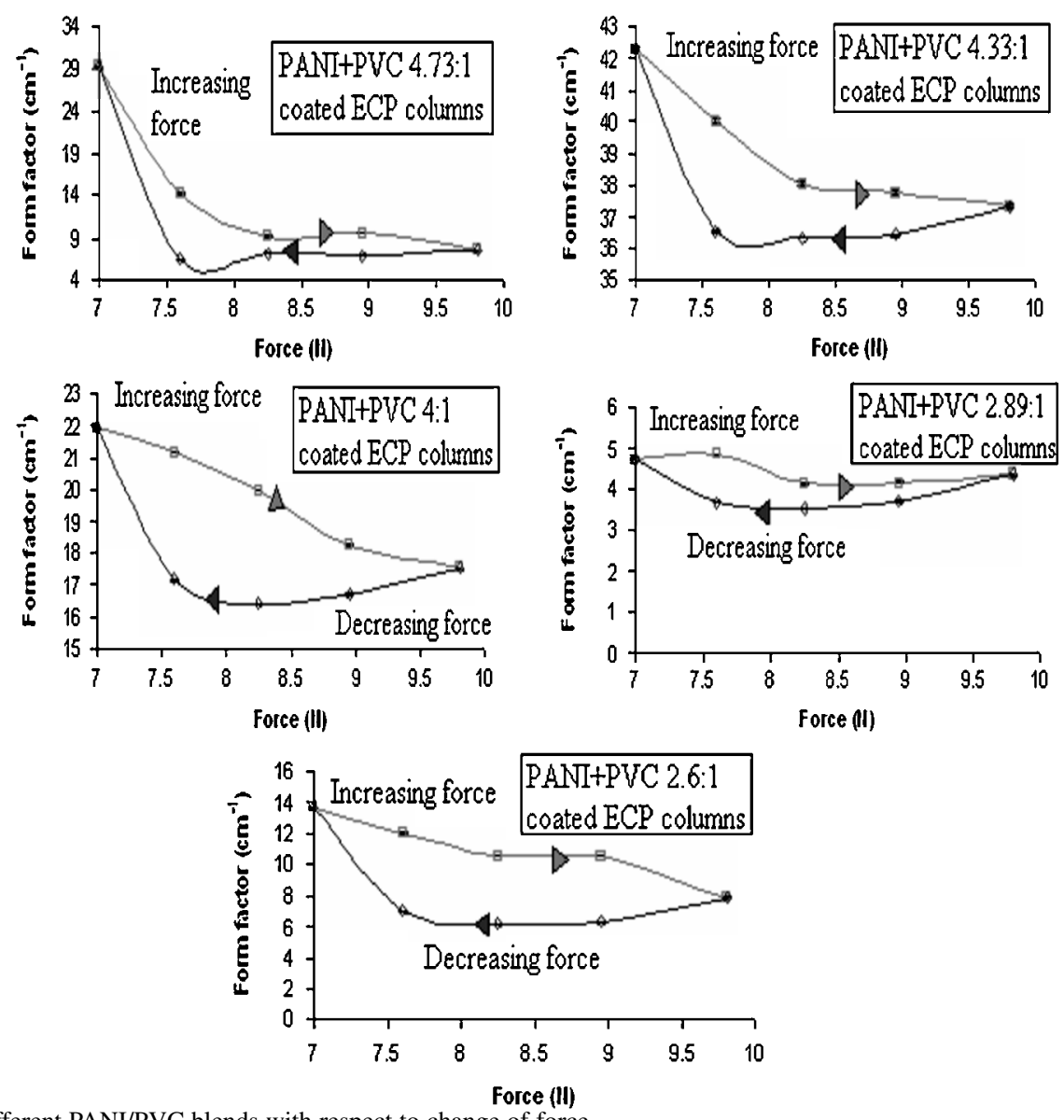

Fig. 14. Form factor of different PANI/PVC blends with respect to change of force.

TABLE IV

MEASUREMENTS OF ICP-COATED PCB TRACK INTERFACE CONFIGURATION

\begin{tabular}{|c|c|c|c|c|c|}
\hline \multirow{2}{*}{$\begin{array}{l}\text { Blend } \\
\text { ratio }\end{array}$} & \multirow{2}{*}{$\begin{array}{c}\operatorname{Arean} \times 10^{5} \\
\left(\mathrm{~cm}^{2}\right)\end{array}$} & \multicolumn{2}{|c|}{$\begin{array}{c}\text { PCB/ICP-ICP/PCB } \\
\text { interface }\end{array}$} & \multicolumn{2}{|c|}{$\begin{array}{l}\text { PCB/ICP-PCB track } \\
\text { interface }\end{array}$} \\
\hline & & $\begin{array}{l}\text { Length } \\
(\mathrm{cm})\end{array}$ & $\begin{array}{c}\text { Resistance } \\
\text { (KS) }\end{array}$ & $\begin{array}{l}\text { Length } \\
\text { (cm) }\end{array}$ & $\begin{array}{c}\text { Resistance } \\
(\mathrm{K} \Omega)\end{array}$ \\
\hline $4.73: 1$ & $\begin{array}{l}7.86 \\
( \pm 0.64 \%)\end{array}$ & $\begin{array}{c}0.030 \\
( \pm 3.33 \%)\end{array}$ & $\begin{array}{c}32.639 \\
( \pm 1.69 \%)\end{array}$ & $\begin{array}{c}0.013 \\
( \pm 7.69 \%)\end{array}$ & $\begin{array}{c}14.143 \\
( \pm 11.66 \%)\end{array}$ \\
\hline 4.33:1 & $\begin{array}{l}7.86 \\
( \pm 0.64 \%)\end{array}$ & $\begin{array}{c}0.030 \\
( \pm 3.33 \%)\end{array}$ & $\begin{array}{c}57.597 \\
( \pm 7.39 \%)\end{array}$ & $\begin{array}{c}0.015 \\
( \pm 6.67 \%)\end{array}$ & $\begin{array}{c}28.798 \\
( \pm 5.38 \%)\end{array}$ \\
\hline 4:1 & $\begin{array}{l}7.86 \\
( \pm 0.64 \%)\end{array}$ & $\begin{array}{c}0.006 \\
( \pm 16.7 \%)\end{array}$ & $\begin{array}{c}13.840 \\
( \pm 8.75 \%)\end{array}$ & $\begin{array}{c}0.003 \\
( \pm 16.7 \%)\end{array}$ & $\begin{array}{c}6.920 \\
( \pm 10.65 \%)\end{array}$ \\
\hline $2.89: 1$ & $\begin{array}{l}7.24 \\
( \pm 0.69 \%)\end{array}$ & $\begin{array}{c}0.005 \\
( \pm 10.0 \%)\end{array}$ & $\begin{array}{c}112.842 \\
( \pm 2.64 \%)\end{array}$ & $\begin{array}{c}0.003 \\
( \pm 16.7 \%)\end{array}$ & $\begin{array}{c}67.705 \\
( \pm 8.35 \%)\end{array}$ \\
\hline $2.6: 1$ & $\begin{array}{l}7.86 \\
( \pm 0.64 \%)\end{array}$ & $\begin{array}{c}0.002 \\
( \pm 25.0 \%)\end{array}$ & $\begin{array}{c}118.631 \\
( \pm 6.53 \%)\end{array}$ & $\begin{array}{c}0.001 \\
( \pm 30.0 \%)\end{array}$ & $\begin{array}{c}59.315 \\
( \pm 2.31 \%)\end{array}$ \\
\hline
\end{tabular}

resistances of the PCB/ICP-ICP/PCB interface were approximately double that of the PCB/ICP-PCB track interface. The contact areas for both configurations did not differ much. The conductivities for the different blend ratios were recalculated and comparing the corresponding values obtained by the PANI/PVC blend-coated ECP columns from Fig. 13, it was noted that the differences were found to be relatively small at approximately $3.6 \%$.

\section{DISCUSSION}

\section{A. PEDOT/PSS and the Derivatives}

The PEDOT/PSS:DMF mixture at 3:1 gave the optimum conductivity value of $7.96 \times 10^{-2} \mathrm{Scm}^{-1}$. When the amount of
DMF was increased beyond this ratio, a sudden decrease in conductivity was noted. The actual reason has not been thoroughly investigated but this could be due to saturation of the derivatives where PEDOT/PSS no longer dominates the electrical characteristics of the overall conducting polymer.

Conversely, the introduction of an exceeded level of DMF could have caused relaxation in the material, which in turn could affect the energy band gap within the polymer, resulting in the lower conductivity levels.

Although conductivity of the 3:1 derivative increased approximately six times that of the undoped PEDOT/PSS, accurate repeatability of the measurement under varying compression forces appeared to be the trade-off. At a constant force, the measurements showed quick transient and stable steady-state response. However, when the compression force was altered then readjusted back to the previous known fixed force, the magnitudes of contact resistances appeared to be offset with deviations ranging from $\pm 2 \%$ and $\pm 25 \%$. This offset was proportional to the increased amount of DMF in the overall ICP mixture.

When subjected to an increasing or decreasing compression force, a reversible material should vary its physical dimensions accordingly. If this phenomenon was not apparent, the material could be consider deformed. Deformation could be due to several reasons such as the loss of materials and the random or irregular defects in the structure when under certain strain or stress levels. In this aspect, repeatable deformation could involve the elastic property of the material. The changes of form factors (length/area ratio) for more highly-doped DMF ICPs 


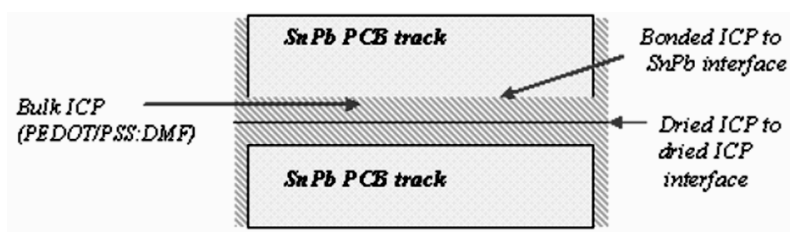

Fig. 15. Layered structure of the ICP-ICP interface.

under varying cyclic force measurements as well as the physical changes in untested and tested samples could suggest the presence of a more elastic material.

For the experiments involving ICP-coated PCB tracks, the conductivities of the PCB/ICP-PCB track interfaced structure were found to be relatively close as compared to that of the ICP-coated ECP columns with an averaged deviation of $16 \%$. This could be due to the estimations made to regressed data as well as the approximated length and area values of the contact interface. For the PCB/ICP-ICP/PCB interfaced structure, two dried ICP surfaces were in contact. The contact resistance would be measured across the structure enclosed between the $\mathrm{SnPb}$ PCB tracks as shown in Fig. 15. This includes two interfaces of bonded ICP with $\mathrm{SnPb}$, two bulk materials of ICPs and a dried ICP to ICP interface at contact point.

Theoretically, the contact resistance measured should be approximately twice that of the PCB/ICP-PCB track interfaced structure. However, the results seemed to differ and the conductivity of the ICP-ICP interfaced structure was approximately $53 \%$ lower than that of the ICP-PCB track interfaced equivalent. These observations could suggest a disruption or nonuniformity of conduction at the unbonded ICP-ICP interface. The reaction involved at such an interface would require further investigations should a contact be fabricated in this manner. The conductivities shown in Figs. 10 and 12 (for ICP-PCB interface) would be more viable and representative results for this initial study of employing PEDOT/PSS and its derivatives as electrical contact and coatings.

\section{B. PANI/PVC Blends}

Original PANI is available in powdered form and practically insoluble in most solvents. Hence, it is almost impossible to coat a layer over surfaces with respectable thickness levels. In order to overcome this disadvantage, PANI is mixed with an insulating polymer to form commodity blends.

From the experiments, the (4.73:1) PANI/PVC blend was found to produce the highest conductivity of $1.17 \times 10^{-2} \mathrm{Scm}^{-1}$. When the amount of PVC (insulating material) increased, conductivity decreased exponentially with improvement in the mechanical structure of the polymer blend. The repeatable results obtained from the samples employing PANI/PVC blend-coated ECP columns and PANI/PVC blend indicated consistency in conductivity and physical changes, with the averaged deviation of $3.6 \%$.

The hystereses formed in the graphs relating the changes of form factors with respect to varying forces suggested that the instantaneous change of form factor took on different routes of deformation under the influences of compression and relaxing forces. Larger hystersis curves were produced in samples with high PVC content. To further investigate and subsequently improve the hysteresis effect, a different type of PVC or insulating material where the deformation due to compression and relaxing forces follows similar responses, should be explored as alternative materials in the commodity blend. Generally, PANI/PVC blend could be considered as a stable and well-behavior conducting polymer with continuity of conduction at interfaces involving different materials.

\section{CONCLUSION}

In this primary study, the optimum conductivity levels for the ICPs of PEDOT/PSS: DMF and PANI/PVC blend were found in the $10^{-2} \mathrm{Scm}^{-1}$ range. Although these values are comparatively low with respect to extrinsic conducting polymers and metals, ICPs have shown initial potentials in conductivity enhancement and possible implementations as an electrical contact or conductive coating.

The nonlinear but relatively repeatable responses depicted in the relationships established between the form factor and the force for the ICPs presented in this paper could suggest deformation due to the elastic property of the polymer. This property could prove useful to minimize the influences of micromovements at contact surfaces by the absorption and dispersion of the kinetic energy through the material. This way, fretting or abrasive effects which are usually found on the surfaces of metallic contacts could be significantly reduced. The tested surfaces did not reveal scratched or wear marks when subjected to compression forces. In order to validate the hypothesis, further fretting tests would be carried out on these materials. In addition, conductivity enhancement would have to be optimized using different developing and fabricating processes for the ICPs. For a more complete evaluation, the behavior of the ICP-coated contacts using the nonlinear method [17] for measuring resistance would be required.

\section{REFERENCES}

[1] M. Aldssi, Intrinsically conducting polymers: An Emerging Technology. Norwell, MA: Kluwer, 1993, vol. 246.

[2] D. R. Lide and H. P. R. Frederikse, CRC Handbook of Chemistry and Physics a Ready-Reference Book of Chemical and Physical Data, 77th ed. Boca Raton, FL: CRC Press, 1996.

[3] L. Alcacer, Conducting Polymers. Amsterdam, The Netherlands: Reidel, 1987.

[4] J. Swingler and J. McBride, "Fretting corrosion studies of an extrinsic conducting polymer and tin interface," in Proc. 47th IEEE Holm Conf. Elect. Contacts, 2001, pp. 215-219.

[5] T. A. Scotheim, R. L. Elsenbaumer, and J. R. Reynolds, Eds., Handbook of Conducting Polymers, 2nd ed. New York: Marcel Dekker, 1998.

[6] J. O. Iroh and W. Su, "Characterization of the passive inorganic interphase and polypyrrole coatings formed on steel by the aqueous electrochemical process," J. Appl. Polym. Sci., vol. 71, no. 12, pp. 2075-2086, 1999.

[7] A. Kaynak, "Electromagnetic shielding effectiveness of galvanostatically synthesized conducting polypyrrole films in the $300-2000 \mathrm{MHz}$ frequency range," Mater. Res. Bull., vol. 31, no. 7, pp. 845-860, 1996.

[8] R. S. Kohlman, A. J. Epstein, T. A. Scotheim, R. L. Elsenbaumer, and J. R. Reynolds, Eds., Handbook of Conducting Polymers, 2nd ed. New York: Marcel Dekker, 1998, vol. 92.

[9] D. F. Shriver, P. W. Atkins, and C. H. Langford, Inorganic Chemistry, 3rd ed. London, U.K.: Oxford Univ. Press, 1999.

[10] A. Pud, N. Ogurtsov, A. Korzhenko, and G. Shapoval, "Some aspects of preparation methods and properties of polyaniline blends and composites with organic polymers," Progr. Polym. Sci., vol. 28, no. 12, pp. 1701-1753, 2003. 
[11] V. E. Gul, Ed., Structure and properties of conducting polymer composites (new concepts in polymer science). Leiden, The Netherlands: VSP, Jun. 1996.

[12] S. van der Ven, "Polypropylene and other polyolefins," in Polymerization and Characterization Studies in Polymer Science. New York: Elsevier, 1990, vol. 7.

[13] J. Y. Kim, J. H. Jung, D. E. Lee, and J. Joo, "Enhancement of electrical conductivity of poly(3,4-ethylenedioxythiophene)/poly(4-styrenesulfonate) by a change of solvents," Synth. Metals, vol. 126, no. 2-3, pp. 311-316, 2002.

[14] AZoM, Inc., "Polyaniline-Processing and Applications," Tech. Rep., [Online] Available: http://www.azom.com/, 2002.

[15] Q. Pei, G. Zuccarello, M. Ahlskog, and O. Inganäs, "Electrochromic and highly stable poly(3,4-ethylenedioxythiophene) switches between opaque blue-black and transparent sky blue," Polymer, vol. 35, no. 7, pp. 1347-1351, 1994.

[16] Tyco Microelectronics, "Metallized Particle Interconnect-Applications guide (Rev. B)," Product Specifications, 2005.

[17] J. W. McBride, "An experimental investigation of worn electrical contacts using nonlinear contact resistance measurement," in Proc. 16th Int. Conf. Elect. Contacts, 1992, pp. 123-128.

Yu-Zhi (Liza) Lam received the B.Eng. and M.Eng. degrees in electrical and electronics engineering from Nanyang Technological University, Singapore, in 1997 and 2000, respectively, and the Ph.D. degree in research and development of the biomedical oxygen sensor from the Thick Film Unit, University of Southampton, Southampton, U.K., in 2003.

She is currently a Research Fellow with the Electromechanical Group, University of Southampton and also lectures at the Faculty of Technology, Southampton Institute. Her research interests include sensors and instrumentation especially in the field of biomedical engineering and MEM/NEM technologies.
Jonathan Swingler received the M.S. degree in physics and chemistry from the University of Keele, U.K., in 1990 and the Ph.D. degree in the degradation of electrical contacts under low frequency fretting conditions from Loughborough University, Loughborough, U.K., in 1994.

Since then, he has been pursuing research in the area of electrical contact science and technology at the University of Southampton and is currently a Senior Research Fellow in the Electromechanical Research Group.

Dr. Swingler is a Chartered Physicist.

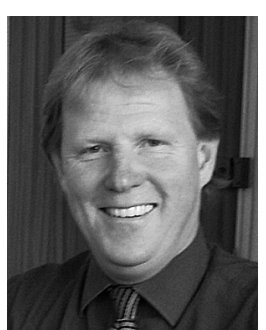

John W. McBride received the M.S. degree in aeronautical engineering from the University of Southampton, Southampton, U.K., in 1978 and the $\mathrm{Ph} . \mathrm{D}$. degree in electrical contact phenomena from Plymouth University, Plymouth, U.K., in 1986.

Since 1987, he has been Lecturer, Senior Lecturer, and now Professor of Electromechanical Engineering in the School of Engineering Science, University of Southampton. He is Chair of the Electromechanical Research Group. His research interests include instrumentation; surface metrology, applied to optics, MEMS, wear analysis, early sound recordings, and electrical contact phenomena.

Dr. McBride is an Associate Editor of the IEEE TRANSACTIONS ON Components And PaCKaging TeChNOLOGIES and a member of the Organizing Committee, IEEE Holm Conference on Electrical Contacts. 